\title{
Variables associated with unplanned general adult ICU admission in hospitalised patients: protocol for a systematic review
}

\author{
James Malycha ${ }^{1 *} \mathbb{D}$, Tim Bonnici $^{1}$, Katarina Sebekova ${ }^{1}$, Tatjana Petrinic ${ }^{2}$, Duncan Young ${ }^{1}$ and Peter Watkinson ${ }^{1}$
}

\begin{abstract}
Background: Failure to promptly identify deterioration in hospitalised patients is associated with delayed admission to intensive care units (ICUs) and poor outcomes. Existing vital sign-based Early Warning Score (EWS) algorithms do not have a sufficiently high positive predictive value to be used for automated activation of an ICU outreach team. Incorporating additional patient data might improve the predictive power of EWS algorithms; however, it is currently not known which patient data (or variables) are most predictive of ICU admission. We describe the protocol for a systematic review of variables associated with ICU admission.

Methods/design: MEDLINE, EMBASE, CINAHL and the Cochrane Library, including Cochrane Database of Systematic Reviews and the Cochrane Central Register of Controlled Trials (CENTRAL) will be searched for studies that assess the association of routinely recorded variables associated with subsequent unplanned ICU admission. Only studies involving adult patients admitted to general ICUs will be included. We will extract data relating to the statistical association between ICU admission and predictor variables, the quality of the studies and the generalisability of the findings.
\end{abstract}

Discussion: The results of this review will aid the development of future models which predict the risk of unplanned ICU admission.

Systematic review registration: PROSPERO: CRD42015029617

\section{Background}

Unplanned admission of a patient in an acute care hospital to an intensive care unit (ICU) is a frequent occurrence [1]. Analysis of the Intensive Care National Audit and Research Centre (ICNARC) database shows that in 2012 roughly 40,000 patients had an unplanned ICU admission with up to $80 \%$ of these patients experiencing a preceding period of unchecked clinical deterioration [2-4]. Their mortality rate was 1.5 times that of patients admitted directly to ICU from the emergency department (30.3\% versus $19.7 \%)$. Timely admission to an ICU may improve outcomes for these patients [5]. Many institutions worldwide use risk scores to trigger escalation in care. Escalation of care

\footnotetext{
* Correspondence: james.malycha@ndcn.ox.ac.uk

${ }^{1}$ Kadoorie Centre for Critical Care Research and Education, John Radcliffe

Hospital, Level 3, Headley Way, Oxford OX3 9DU, UK

Full list of author information is available at the end of the article
}

based on an Early Warning Score (EWS) is mandated in the UK [6].

Despite implementation of EWS systems, missed clinical deterioration remains a significant problem [7]. Cognitive errors and barriers to communication have been identified as causes of missed deterioration [8]. In an attempt to bypass these problems, some institutions have trialled directly linking electronic vital sign charts to alerting systems [9]. However, existing EWS algorithms, which are typically based on vital signs, have a poor positive predictive value for severe deterioration [10]. Therefore, they cannot be usefully deployed in systems which aim to automatically alert trained specialists to impending deterioration on the ward as the number of false alerts is excessive. Inclusion of additional variables can improve the accuracy of EWS models $[11,12]$. 


\section{Objective}

We will conduct a systematic review to identify studies of patient-derived variables that are associated with an increased risk of unplanned ICU admission. For the purposes of the review, a variable is defined as an indivisible entity, as opposed to a composite entity such as a risk score, which is made up of multiple variables. A patient-derived variable is a measure of the properties of a patient as opposed to a measure of institutional processes such as nurse-to-patient ratio or number of escalation calls.

\section{Methods/design}

This protocol will adhere to the requirements of Preferred Reporting Items for Systematic Reviews and Meta-analyses Protocol (PRISMA-P), which is included as Additional file 1.

\section{Search strategy}

Papers will be identified by searching Medical Literature Analysis and Retrieval System Online (MEDLINE), Excerpta Medica database (EMBASE), Cumulative Index to Nursing and Allied Health Literature (CINAHL), the Cochrane Database of Systematic Reviews and the Cochrane Central Register of Controlled Trials (CENTRAL). We will include additional papers from the references of reviews articles or studies identified during screening and papers from the authors' personal libraries. A full description of the search strategy is outlined in Appendix 1.

\section{Study selection}

Two researchers will independently screen titles and abstracts of identified papers against the inclusion and exclusion criteria. They will not be blinded to the journal titles or to the study authors or institutions. If there is disagreement or uncertainty regarding eligibility, the article will be included in the next stage of screening for further analysis for inclusion/exclusion. The full text will be retrieved for all articles not excluded by the initial screening. These papers will be independently assessed against the inclusion and exclusion criteria. Disagreements about eligibility will be resolved by discussion between the screening researchers or a third party.

\section{Inclusion criteria}

\section{Types of studies}

Quantitative studies published in peer reviewed journals assessing adults admitted to adult hospitals will be eligible for inclusion in this review. Studies will most likely be prospective or retrospective cohort and casecontrol studies.

\section{Study characteristics}

Eligible studies must include both a cohort of patients admitted to ICU and a cohort not admitted to ICU. Unplanned ICU admission may be either a primary or secondary outcome measure. Studies published from January 2000 until the day of search completion will be included to ensure modern day applicability. No language restrictions will be applied.

\section{Phenomenon of interest}

Studies must describe a statistical relationship between a patient-derived variable (e.g. heart rate or creatinine level) and an unplanned admission to intensive care from a general ward or emergency department. 'Diagnosis' or 'groups of diagnoses' are eligible to be included as variables. If a paper analyses both eligible variables (e.g. variables that are widely available in most UK hospitals) and non-eligible variables (e.g. variables that are not widely available in most UK hospitals), it will still be eligible for inclusion, with the authors using only the eligible variables for inclusion in the review.

\section{Population}

Studies that sample adult patients with an unplanned admission to ICU will be considered for inclusion. For the purpose of this review, adult is defined as $>16$ years of age. There will be no other restrictions.

\section{Exclusion criteria \\ Types of studies}

Qualitative studies, case studies, grey-literature, editorials, letters, practice guidelines and abstract-only reports will be excluded.

\section{Study characteristics}

Studies of cohorts defined by a single condition or narrow group of conditions (e.g. trauma or sepsis) will be excluded. We will also exclude studies that do not use a control versus intervention group.

\section{Phenomenon of interest}

Studies of ICU readmission or admission to ICUs dedicated to narrow cohorts of patients will be excluded (e.g. patients admitted to ICU with acute liver failure).

\section{Population}

Studies of participants under 16 years old will be excluded.

\section{Data extraction}

Two authors will independently extract data from the papers and supplementary material. All uncertainties regarding data extraction will be resolved by discussion amongst the study team. DistillerSR (Evidence Partners, Ottawa, Canada) will be used to manage the data and 
identify duplicate search results. All screening and data extraction forms will be implemented within DistillerSR. As part of the development of this protocol the study forms have been piloted and a calibration exercise has been undertaken to ensure good interrater agreement.

\section{Quality assessment}

Risk of bias will be assessed using a scoring system adapted from two previous systematic reviews, [13, 14] both of which are adapted from the Newcastle-Ottawa Scale (NOS) [15]. The NOS is a scoring system designed to assess the quality of nonrandomised studies in metaanalyses. Using a 'star' system, it attributes a score to a paper after assessing the selection of study groups, the comparability of the groups and the ascertainment of either the exposure or outcome of interest for casecontrol or cohort studies. The scoring system used in this systematic review is outlined in Appendix 2.

\section{Data synthesis and analysis}

We will conduct a qualitative synthesis of results from included studies. This will be presented descriptively in table and text form. We will extract summary comparison data as odds ratios (95\% confidence intervals) where possible. Where sufficient original data is presented we will calculate odds ratios. Where insufficient data is presented we will contact the authors.

\section{Discussion}

As hospitals move towards fully digital patient records, increasing amounts of data are being collected in hospital Clinical Information Systems. Researchers have begun using this resource to develop models to predict patient deterioration based upon electronically captured data $[16,17]$. These models are reported to perform better than conventional EWS algorithms but their clinical adoption is not widespread.

Commonly, patient deterioration prediction models aim to accurately predict one of cardiac arrest, death or unplanned ICU admission. This systematic review will be the first to bring together the hospitalised patient factors that are known to be associated with subsequent urgent admission to ICU alone. This is a vital step in starting to use this information to identify patients at risk of ICU admission.

The findings from this review will contribute to the construction of an improved model for the prediction of clinical deterioration and unplanned ICU admission in adult patients on general wards. The findings may also be useful for researchers seeking to improve upon existing work in this field.

\section{Appendix 1}

Table 1 Draft search strategy for MEDLINE

1. (ICU* OR "intensive care" OR "critical care").ab,ti.
2. INTENSIVE CARE UNITS/
3. 1 OR 2
4. (admission* OR admitted OR transfer").ab,ti.
5. ("risk assessment*" OR "risk facto**" OR "risk stratif*" OR predict* OR "increased risk"
OR trigger* OR score* OR scoring OR "early warning"
OR escalat" OR deteriorat" OR triag* OR "vital sign*" OR model* OR validat*).ab,ti.
6. 3 AND 4 AND 5
7. limit 6 to (humans AND yr = "2000 Current")
8. (observational OR "case control*" OR retrospective OR cohort*
OR "systematic review").ab,ti.
9. OBSERVATIONAL STUDY/
10. CASECONTROL STUDIES/
11. RETROSPECTIVE STUDIES/
12. COHORT STUDIES/
13. RANDOMIZED CONTROLLED TRIAL/
14. REVIEW/
15. COMPARATIVE STUDY/
16. PROSPECTIVE STUDIES/
17. VALIDATION STUDIES/
18. 8 OR 9 OR 10 OR 11 OR 12 OR 13 OR 14 OR 15 OR 16 OR 17
19. 7 AND 18
20. (Unplanned OR unexpected OR unanticipated OR emergency OR "rapid response").ab,ti.
21. 19 AND 20

\section{Appendix 2}

Table 2 Risk of bias scoring

\begin{tabular}{ll}
\hline Participant selection & Score \\
\hline Cohort studies & 2 \\
$\begin{array}{l}\text { Selected cohort is very representative of the general hospitalised population } \\
\text { Selected cohort is somewhat representative of the general hospitalised }\end{array}$ & 1 \\
population & \\
$\begin{array}{l}\text { Cohort is not representative of the general hospital population or the selection } \\
\text { of the group was not described }\end{array}$ & 0 \\
Case-control studies & 2 \\
$\begin{array}{l}\text { Cases and controls drawn from the same population and population is very } \\
\text { representative of the general hospitalised population }\end{array}$ & 2 \\
$\begin{array}{l}\text { Cases and controls drawn from the same population and population is } \\
\text { somewhat representative of the general hospitalised population }\end{array}$ & 1 \\
$\begin{array}{l}\text { Cases and controls drawn from different sources or the } \\
\text { selection of groups was not described }\end{array}$ & 0 \\
Comparability of groups & 2 \\
No differences between the groups explicitly reported unless it was one of \\
these variables that was under investigation, or such differences were \\
$\begin{array}{l}\text { adjusted for } \\
\text { Differences between groups were not recorded } \\
\text { Groups differed }\end{array}$ \\
$\begin{array}{l}\text { Size } \\
\text { <100 participants in each group participants in each group }\end{array}$ \\
$\begin{array}{l}\text { Adjustment for confounding } \\
\text { No adjustment for cofounders }\end{array}$ \\
\hline
\end{tabular}




\section{Additional file}

Additional file 1: PRISMA-P (Preferred Reporting Items for Systematic review and Meta-Analysis Protocols) 2015 checklist: recommended items to address in a systematic review protocol. (DOC $97 \mathrm{~kb}$ )

\section{Abbreviations}

CENTRAL: The Cochrane Database of Systematic Reviews and the Cochrane Central Register of Controlled Trials; CINAHL: Cumulative Index to Nursing and Allied Health Literature; EMBASE: Excerpta Medica database; EWS: Early Warning Score; ICU: Intensive care unit; MEDLINE: Medical Literature Analysis and Retrieval System Online; NICE: National Institute of Clinical and Healthcare Excellence

\section{Acknowledgements}

This work has been funded by a Wellcome Trust (HICF-R9-524), Department of Health and the National Institute for Health Research (NIHR) Oxford Biomedical Research Centre Programme. All views expressed are those of the authors and not necessarily those of the Wellcome Trust, Department of Health or NIHR.

\section{Funding}

This work has been funded by a Wellcome Trust grant. (HICF-R9-524) and the NIHR Biomedical Research Centre (Oxford). These funders played no role in developing the review.

\section{Availability of data and materials}

Not applicable.

\section{Authors' contributions}

$\mathrm{JM}, \mathrm{TB}, \mathrm{PW}$ and DW have substantially contributed to the design of the systematic review. TP has developed the search strategy. JM, TB, KS, DY and PW have written this manuscript. All authors read and approved the final manuscript. The funders have not been involved in the study design or reporting. JM is guarantor of this review.

\section{Competing interests}

The authors declare that they have no competing interests.

\section{Consent for publication}

Not applicable.

\section{Ethics approval and consent to participate}

Not applicable.

\section{Publisher's Note}

Springer Nature remains neutral with regard to jurisdictional claims in published maps and institutional affiliations.

\section{Author details}

${ }^{1}$ Kadoorie Centre for Critical Care Research and Education, John Radcliffe Hospital, Level 3, Headley Way, Oxford OX3 9DU, UK. ²University of Oxford, Bodleian Health Care Libraries, Academic Centre, John Radcliffe Hospital, Level 3, Headley Way, Oxford OX3 9DU, UK.

Received: 24 May 2016 Accepted: 14 March 2017

Published online: 28 March 2017

\section{References}

1. Adult Critical Care in England - April 2010 to March 2011: Experimental Statistics. In: Hospital Episode Statistics. NHS Digital. http://content.digital. nhs.uk/catalogue/PUB06193/adul-crit-care-data-eng-apr-10-mar-11-rep.pdf. Accessed 27 Mar 2017

2. Kause J, Smith GB, Prytherch DR, Parr M, Flabouris A, Hillman KM, et al. A comparison of antecedents to cardiac arrests, deaths and emergency intensive care admissions in Australia and New Zealand, and the United Kingdom—the ACADEMIA study. Resuscitation. 2004;62:275-82.
3. Stelfox HT, Bagshaw SM, Gao S. Characteristics and outcomes for hospitalized patients with recurrent clinical deterioration and repeat medical emergency team activation. Crit Care Med. 2014;42:1601-9.

4. Buist MD, Bernard S, Nguyen TV, Moore G, Anderson J. Association between clinically abnormal observations and subsequent in-hospital mortality: a prospective study. Resuscitation. 2004;62:137-41.

5. Liu V, Kipnis P, Rizk NW, Escobar GJ. Adverse outcomes associated with delayed intensive care unit transfers in an integrated healthcare system. J Hosp Med. 2012;7:224-30. Wiley Subscription Services, Inc., A Wiley Company.

6. National Institute of Clinical Excellence. In: NICE, editor. Acutely ill patients in hospital. 2007. p. 1-107. Report No.: NCG50.

7. Findlay GP, Shotton H, Kelly K, Mason M. Time to intervene? NCEPOD. National Confidential Enquiry into Patient Outcome and Death (NCEPOD). 2012.

8. Shearer B, Marshall S, Buist MD, Finnigan M, Kitto S, Hore T, et al. What stops hospital clinical staff from following protocols? An analysis of the incidence and factors behind the failure of bedside clinical staff to activate the rapid response system in a multi-campus Australian metropolitan healthcare service. BMJ Qual Saf. 2012;21:569-75.

9. Jones S, Mullally M, Ingleby S, Buist MD, Bailey M, Eddleston JM. Bedside electronic capture of clinical observations and automated clinical alerts to improve compliance with an Early Warning Score protocol. Crit Care Resusc. 2011;13:83-8.

10. Romero-Brufau S, Huddleston JM, Naessens JM, Johnson MG, Hickman J, Morlan BW, et al. Widely used track and trigger scores: are they ready for automation in practice? Resuscitation. 2014;85:549-52.

11. Churpek MM, Yuen TC, Winslow C, Robicsek AA, Meltzer DO, Gibbons RD, et al. Multicenter development and validation of a risk stratification tool for ward patients. Am J Respir Crit Care Med. 2014;190:649-55.

12. Rothman MJ, Rothman SI, Beals J. Development and validation of a continuous measure of patient condition using the Electronic Medical Record. J Biomed Inform. 2013;46:837-48.

13. Taggart DP, D'Amico R, Altman DG. Effect of arterial revascularisation on survival: a systematic review of studies comparing bilateral and single internal mammary arteries. Lancet. 2001;358(9285):870-5.

14. Duckitt $\mathrm{K}$, Harrington D. Risk factors for pre-eclampsia at antenatal booking: systematic review of controlled studies. BMJ. 2005;330:565.

15. Wells G, Shea B, O'Connell D, Peterson J, Welch V, Losos M, Tugwell P. The Newcastle-Ottawa Scale (NOS) for assessing the quality of nonrandomised studies in meta-analyses. 2013.

16. Churpek MM, Yuen TC, Park SY, Meltzer DO, Hall JB, Edelson DP. Derivation of a cardiac arrest prediction model using ward vital signs. Crit Care Med. 2012:40:2102-8

17. Escobar GJ, LaGuardia JC, Turk BJ, Ragins A, Kipnis P, Draper D. Early detection of impending physiologic deterioration among patients who are not in intensive care: development of predictive models using data from an automated electronic medical record. J Hosp Med. 2012;7:388-95.

\section{Submit your next manuscript to BioMed Central and we will help you at every step:}

- We accept pre-submission inquiries

- Our selector tool helps you to find the most relevant journal

- We provide round the clock customer support

- Convenient online submission

- Thorough peer review

- Inclusion in PubMed and all major indexing services

- Maximum visibility for your research

Submit your manuscript at www.biomedcentral.com/submit
Biomed Central 